\title{
Usefulness of a new anthropometric indicator - VAI (Visceral Adiposity Index) in the evaluation of metabolic and hormonal disorders in women with polycystic ovary syndrome
}

\author{
Anna Brończyk-Puzońn ', A, D, E, Paweł Jagielski2, B, C, E, Karolina Kulik-Kupka ${ }^{1, D, E}$, Aneta Koszowska ${ }^{1, D, E}$, \\ Justyna Nowak ${ }^{1}, \mathrm{D}, \mathrm{E}$, Barbara Zubelewicz-Szkodzińska ${ }^{1, A, E, F}$ \\ ${ }^{1}$ Department of Nutrition-Related Diseases Prevention, School of Public Health in Bytom, Medical University of Silesia, Katowice, Poland \\ ${ }^{2}$ Human Nutrition Department, Faculty of Health Science, Jagiellonian University Medical College, Kraków, Poland \\ A - research concept and design; $\mathrm{B}$ - collection and/or assembly of data; $\mathrm{C}$ - data analysis and interpretation; \\ $D$ - writing the article; $E$ - critical revision of the article; $F$ - final approval of article
}

Address for correspondence

Anna Brończyk-Puzoń

E-mail:anna.puzon@op.pl

\section{Funding sources}

None declared

Conflict of interest

None declared

Received on September 20, 2015

Revised on 0ctober 26, 2015

Accepted on December 22, 2015

DOI

\begin{abstract}
Background. Visceral adiposity index (VAI) is a new anthropometric indicator that makes it possible to define the risk of obesity-related cardiometabolic complications even before the diagnosis of metabolic syndrome.
\end{abstract}

Objectives. Evaluating the usefulness of VAI in order to differentiate metabolically unhealthy women (MU-PCOS) and defining the usefulness of this index in everyday practice.

Material and methods. A prospective study was conducted among 43 women who met the Rotterdam criteria for diagnosing polycystic ovarian syndrome. Body composition was measured using bioelectrical impedance analysis. Statistical analysis was performed using STATISTICA v. 10 and relevant statistical tests. The adopted significance level was $p=0.05$.

Results. Based on the study results, a significant positive correlation was found between the value of VAl and the concentration of insulin ( $r=0.33 ; p<0.01)$, HOMA index ( $r=0.46 ; p<0.01)$ and FAl $(r=0.54$; $p<0.01$ ). It was also shown that there is a significant negative correlation between VAl and the concentration of SHBG $(r=-0.53 ; p<0.01)$ and HDL cholesterol $(r=-0.88 ; p<0.01)$. In the group of metabolically unhealthy women, the research showed a significantly higher value of free testosterone, SHGB, DHEAS and FAI $(p<0.05)$.

Conclusions. Visceral adiposity index makes it possible to introduce early prevention of metabolic disorders (including cardiometabolic disorders), as well as to evaluate the increase of hyperandrogenemia in women with polycystic ovary syndrome. The use of the cut-off point of VAI $=1.675$ is a simple way to evaluate women with MU-PCOS.

Key words: polycystic ovarian syndrome, visceral adiposity index, metabolic disorders

10.17219/acem/61100

Copyright

Copyright by Author(s)

This is an article distributed under the terms of the

Creative Commons Attribution Non-Commercial License

(http://creativecommons.org/licenses/by-nc-nd/4.0/) 


\section{Background}

Visceral adiposity index (VAI) is a recent anthropometric indicator indirectly reflecting the risk of obesityrelated cardiometabolic complications. Its application allows early evaluation of such risk, even prior to diagnosing overt metabolic syndrome. ${ }^{1}$ VAI can be considered a marker of adipose tissue dysfunction as it provides reliable data when used in the general population and in patients with polycystic ovary syndrome, acromegaly, type 2 diabetes, viral hepatitis $C$, non-alcoholic fatty liver diseases. ${ }^{1}$ This index allows accurate identification of visceral adipose tissue in women with polycystic ovary syndrome, determining intra-abdominal adipose tissue content in a similar way to a computed tomography (CT) scan performed in this group of female patients. ${ }^{2} \mathrm{VAI}$ is an empirical-mathematical model, gender-specific and based on anthropometric measurements, such as BMI and waist circumference, and biochemical parameters, namely triglyceride (TG) and high-density lipoprotein cholesterol (HDL-C) concentrations. ${ }^{3}$

Fig. 1 illustrates the formula of the visceral adiposity index for women.

Fig.1. Waist circumference $(\mathrm{cm}), \mathrm{BMl}\left(\mathrm{kg} / \mathrm{m}^{2}\right)$, TG $(\mathrm{mmol} / \mathrm{L}), \mathrm{HDL}(\mathrm{mmol} / \mathrm{L})$

$\mathrm{VAI}=\left(\frac{\text { waist circumference }}{36.58+(1.86 \times \mathrm{BMI})}\right) \times\left(\frac{\mathrm{TG}}{0.81}\right) \times\left(\frac{1.52}{\mathrm{HDL}}\right)$

Polycystic ovary syndrome (PCOS) manifests itself in menstrual disorders, clinical and biochemical hyperandrogenism and polycystic ovaries on ultrasound. In addition, it is also responsible for higher risk of cardiovascular diseases, impaired glucose tolerance and type 2 diabetes. ${ }^{4}$ Insulin resistance and android (abdominal) obesity in PCOS women are not always associated with increased BMI. Excess body weight is not necessary for increased cardiometabolic risk to occur in this group of patients, however it leads to the exacerbation of clinical symptoms of the syndrome in question. ${ }^{5}$ Since VAI is a simple and easy method for evaluation of cardiometabolic risk, it can be a useful tool for the assessment of metabolic risk related to visceral obesity both in everyday medical practice and in population studies. Values of VAI > 1.675 make it possible to differentiate women with metabolically unhealthy polycystic ovary syndrome (MU-PCOS) from women with metabolically healthy polycystic ovary syndrome (MH-PCOS). Nevertheless, the index should not be used for individuals who are under 16 years of age, who have morbid obesity (BMI $>40 \mathrm{~kg} / \mathrm{m}^{2}$ ), who are diagnosed with pendulous abdominal skin folds or hypertriglyceridemia, nor in subjects who are being treated with fibrates and/or are following a low-energy diet. ${ }^{1}$

\section{Objectives}

Application of the cut-off point for VAI $=1.675$ in order to distinguish women with metabolically unhealthy polycystic ovary syndrome (MU-PCOS) and to determine the usefulness of the index in question among women diagnosed with polycystic ovary syndrome in everyday clinical practice.

\section{Material and methods}

The prospective study was conducted among 43 women hospitalized at the Department of Endocrinology and Gynecology of Silesian University, and fulfilling the Rotterdam criteria for diagnosing polycystic ovary syndrome. The diagnosis of the syndrome had to include at least 2 of the following 3 criteria: oligoovulation or anovulation, clinical and/or biochemical hyperandrogenism, polycystic ovaries on ultrasound after exclusion of other causes of the above-mentioned symptoms. The exclusion criteria applied in the study were: diagnosis of androgen excess disorders (congenital or late-onset congenital adrenal hyperplasia, androgen-secreting tumors, Cushing's disease/syndrome, hyperprolactinemia, idiopathic hirsutism), uncontrolled thyroid disorders, mental disorders, morbid obesity $\left(\right.$ BMI $\left.>40 \mathrm{~kg} / \mathrm{m}^{2}\right)$, pendulous abdominal skin folds, hypertriglyceridemia, low-energy diet, use of hormonal contraception in the past 6 months, taking fibrates or steroids, and being over the age of 40 .

Prior to conducting the actual studies, the consent of the Bioethics Committee was obtained, no. KNW/0022/ KB1/53/14, dated June $3^{\text {rd }}$, 2014. Body composition was evaluated by bioelectrical impedance analysis utilizing a Tanita BC-420 MA Body Composition Analyzer (product in accordance with the Medical Device Directive MDD 93/42 EEC). The standard anthropometric measurement method was applied for the circumference of the waist, hips, wrist, shoulder and calf. A stadiometer (Tanita HR-100 Height Rod) was used to measure body height. Waist-to-hip ratio (WHR) was adopted in the study. It can be calculated by dividing the waist circumference (in $\mathrm{cm}$ ) at the umbilical level by the hip circumference (in $\mathrm{cm}$ ) at the superior iliac spine level. Values higher than or equal to 0.85 indicate abdominal obesity and lower than 0.85 gluteal-femoral obesity. The value of waist-to-height ratio (WHtR) was obtained by dividing the waist circumference (in $\mathrm{cm}$ ) at the umbilical level by the body height (in $\mathrm{cm}$ ). Results higher than 0.5 indicate increased risk of obesity-related cardiovascular diseases.

Blood samples were collected in the morning during routine patient examinations in the ward. The tests were performed by means of the immunoenzymatic method (ELISA) using commercial test kits in an accredited analytical laboratory monitored by the external quality as- 
surance programs. Laboratory blood tests included complete lipidogram, levels of glucose, total testosterone, free testosterone, sex hormone binding globulin (SHBG), dehydroepiandrosterone (DHEAS), insulin, homeostatic model assessment of insulin resistance (HOMA-IR), and free androgen index (FAI).

The studied women were divided into 2 groups with metabolically healthy polycystic ovary syndrome $(\mathrm{MH}-$ PCOS; VAI < 1.675) and metabolically unhealthy polycystic ovary syndrome (MU-PCOS; VAI > 1.675). The statistical analysis of the obtained results was conducted with STATISTICA v. 10.0. The Shapiro-Wilk test was utilized to verify normal distribution for the analyzed variables. Statistical tests to verify particular study hypotheses were properly selected in order to reveal differences or correlations between the variables. For the differences between the study groups to be determined, the following tests were applied: the Student's t-test (normal distribution) and the Mann-Whitney U-test (abnormal distribution). The results showing normal distribution were expressed as an average value $(\mathrm{x}) \pm$ standard deviation (SD). The parameters with abnormal distribution were expressed as median and interquartile ranges. For assessment of the correlations between the analyzed variables, the Spearman rank correlation was used. The significance level of $\mathrm{p}=0.05$ was adopted.

\section{Results}

The study was carried out in a group of women at the age of $18-38(26.52 \pm 5.73)$. The average values of the selected anthropometric parameters were as follows: body weight $73.54 \pm 19.07 \mathrm{~kg}$; waist circumference $87.07 \pm 16.58 \mathrm{~cm}$; BMI $27.16 \pm 6.91 \mathrm{~kg} / \mathrm{m}^{2}$; WHR $0.82 \pm 0.08$; WHtR $0.53 \pm 0.1$; fat mass percentage (FM\%) 34.01 \pm 9.7. Table 1 illustrates the detailed characteristics of the MH-PCOS and MU-PCOS women.

Table 1. Comparison of data from patient groups before and after the operation

\begin{tabular}{|l|c|c|c|}
\hline \multicolumn{1}{|c|}{ Parameters } & MH-PCOS $(n=28)$ & MU-PCOS $(n=15)$ & p-value \\
\hline Age (years) & $26(22.5-31)$ & $25.27 \pm 5.57$ & 0.3021 \\
\hline Body weight (kg) & $61.3(54.9-70.25)$ & $89.8(74-103.2)$ & $<0.01$ \\
\hline $\begin{array}{l}\text { Waist } \\
\text { circumference } \\
(\mathrm{cm})\end{array}$ & $79.52 \pm 11.9$ & $100.5 \pm 16.03$ & $<0.01$ \\
\hline BMI (kg/m²) & $22.85(20.7-25.25)$ & $32.61 \pm 6.44$ & $<0.01$ \\
\hline WHR & $0.78 \pm 0.06$ & $0.89 \pm 0.08$ & $<0.01$ \\
\hline WHtR & $0.47(0.43-0.52)$ & $0.62 \pm 0.09$ & $<0.01$ \\
\hline FM\% & $29.77 \pm 7.98$ & $42.9(37-46.1)$ & $<0.01$ \\
\hline
\end{tabular}

FM\% - fat mass percentage.
Significant differences were revealed between the study groups of women, which concerned the average values of the following anthropometric parameters and indicators: body weight, waist circumference, BMI, WHR, WhtR and $\mathrm{FM} \%$. The average values of the analyzed tests were significantly higher in the MU-PCOS group than in the MH-PCOS group $(\mathrm{p}<0.01)$.

The study also revealed significant differences between the $\mathrm{MH}-\mathrm{PCOS}$ and MU-PCOS groups with regard to the average values of the following biochemical studies: concentrations of triglycerides, HDL cholesterol, SHBG, free testosterone, DHEAS and HOMA-IR ( $<$ 0.05) (Table 2).

Table 2. Analysis of the differences of the average values of the selected biochemical studies between the groups of MH-PCOS and MU-PCOS women

\begin{tabular}{|c|c|c|c|}
\hline Parameters & $\mathrm{MH}-\mathrm{PCOS}(\mathrm{n}=28)$ & MU-PCOS $(n=15)$ & $p$-value \\
\hline $\begin{array}{l}\text { Testosterone } \\
(\mathrm{nmol} / \mathrm{L})\end{array}$ & $2.01 \pm 0.73$ & $2.15(1.63-3.12)$ & 0.1327 \\
\hline $\begin{array}{l}\text { Free } \\
\text { testosterone } \\
\text { (pmol/L) }\end{array}$ & 15.19 (11.13-30.93) & $32.66(17.86-44.83)$ & $<0.05$ \\
\hline $\begin{array}{l}\text { SHBG } \\
(\mathrm{nmol} / \mathrm{L})\end{array}$ & $60 \pm 29.54$ & $27.70(18.25-52.60)$ & $<0.05$ \\
\hline $\begin{array}{l}\text { DHEAS } \\
\text { (umol/L) }\end{array}$ & $8.36 \pm 3.41$ & $10.82 \pm 3.21$ & $<0.05$ \\
\hline FAl & $3.3(3.1-5.5)$ & $6.65(4.8-12.75)$ & $<0.05$ \\
\hline $\begin{array}{l}\text { Glucose } \\
\text { (mmol/L) }\end{array}$ & $5.0(4.67-5.22)$ & $5.25 \pm 0.5$ & 0.0647 \\
\hline $\begin{array}{l}\text { Insulin } \\
\text { (pmol/L) }\end{array}$ & $65.65(43.05-87.54)$ & $85.31 \pm 37.02$ & 0.2082 \\
\hline HOMA & $1.66(1.0-2.5)$ & $2.72 \pm 1.21$ & $<0.05$ \\
\hline $\mathrm{TC}(\mathrm{mmol} / \mathrm{L})$ & $4.84 \pm 0.63$ & $4.56 \pm 0.67$ & 0.1729 \\
\hline $\begin{array}{l}\mathrm{HDL}-\mathrm{C} \\
(\mathrm{mmol} / \mathrm{L})\end{array}$ & $1.83 \pm 0.39$ & $1.16(1.09-1.27)$ & $<0.01$ \\
\hline $\begin{array}{l}\text { LDL-C } \\
(\mathrm{mmol} / \mathrm{L})\end{array}$ & $2.64 \pm 0.66$ & $2.56 \pm 0.68$ & 0.7064 \\
\hline TG (mmol/L) & $0.81 \pm 0.26$ & $1.6(1.37-2.03)$ & $<0.01$ \\
\hline
\end{tabular}

TC - total cholesterol.

The study involved a correlation analysis between VAI and the studied variables for the whole PCOS group $(\mathrm{n}=43)$. The analysis showed a positive correlation between the VAI value and body weight $(\mathrm{r}=0.69 ; \mathrm{p}<0.01)$, WHR ( $\mathrm{r}=0.67$; $\mathrm{p}<0.01)$, WHtR $(\mathrm{r}=0.72 ; \mathrm{p}<0.01)$ and visceral adipose tissue $(\mathrm{r}=0.69 ; \mathrm{p}<0.01)$, as well as FM\% $(\mathrm{r}=0.69 ; \mathrm{p}<0.01)$. The correlation analysis was also performed for biochemical studies (Table 3).

It was found that, along with an increase in the VAI value, the concentrations of insulin, triglycerides, HOMA-IR and FAI also significantly increased ( $\mathrm{p}<0.05)$. A negative correlation was reported between VAI and serum HDL cholesterol and SHGB concentrations $(\mathrm{p}<0.01)$. No significant 
Table 3. Evaluation of the correlations between the VAI value and the selected biochemical studies in the study groups of women $(n=43)$

\begin{tabular}{|l|c|c|}
\multicolumn{1}{|c|}{ VAl } & $\mathrm{R}^{*}$ & p-value \\
\hline Glucose $(\mathrm{mmol} / \mathrm{L})$ & 0.14 & 0.3690 \\
\hline Insulin $(\mathrm{pmol} / \mathrm{L})$ & 0.33 & $<0.05$ \\
\hline TC (mmol/L) & -0.13 & 0.4016 \\
\hline HDL-C (mmol/L) & -0.88 & $<0.01$ \\
\hline LDL-C (mmol/L) & 0.09 & 0.5878 \\
\hline TG (mmol/L) & 0.94 & $<0.01$ \\
\hline SHBG (nmol/L) & -0.53 & $<0.01$ \\
\hline Testosterone (nmol/L) & 0.20 & 0.2053 \\
\hline Free testosterone (pmol/L) & 0.30 & 0.0507 \\
\hline DHEAS (umol/L) & 0.30 & 0.0551 \\
\hline HOMA & 0.46 & $<0.01$ \\
\hline FAl & 0.54 & $<0.01$ \\
\hline
\end{tabular}

*Spearman's rank correlation.

correlations were revealed between VAI and the concentrations of glucose, complete cholesterol, LDL cholesterol, testosterone, free testosterone and DHEAS ( $\mathrm{p}>0.05)$.

\section{Discussion}

VAI, similarly to a computed tomography scan, is an effective indicator of visceral adipose tissue. This was concluded by Jee-Young Oh et al., who had assessed the correlation between VAI and insulin resistance in a group of 180 Korean women with polycystic ovary syndrome. The study revealed a positive correlation between visceral fat area (VFA), measured by a CT scan, and VAI $(\mathrm{r}=0.57 ; \mathrm{p}<0.01) .^{2}$ The authors of this manuscript found significant dependence between VAI and the indicator of visceral adipose tissue measured by the bioelectrical impedance analysis. In another study, which comprised a group of 193 women with PCOS, Androulakis II et al. observed a significant positive correlation between VAI and the concentrations of insulin $(\mathrm{r}=0.57$; $\mathrm{p}<0.01)$, SHBG $(\mathrm{r}=-0.22 ; \mathrm{p}<0.05)$ and HOMA-IR $(\mathrm{r}=0.55, \mathrm{p}<0.01)$. The same observation was made by the authors of this paper. The authors of the quoted study revealed significantly higher values of VAI and HOMA in the group of PCOS women with menstrual disorders versus the female group with normal ovulation. ${ }^{7}$ In the research conducted by the authors of this manuscript, the studied women were not divided according to menstrual disorders.
Amato MC et al., relying on studies conducted in a group of 224 women with PCOS, found that in addition to the fasting plasma glucose level, VAI value and DHEAS concentration can also be considered useful in determining the risk of diabetes in the screening tests for all women with polycystic ovary syndrome. ${ }^{8}$ The authors of this manuscript did not reveal a significant dependence between VAI and the fasting plasma glucose level. Such a correlation, however, was found between insulin and HOMA concentrations $(p<0.05)$. Moreover, a correlation analysis between VAI and FAI was performed. On the basis of the results obtained, it can be claimed that the index in question is also an effective tool in assessing the severity of hyperandrogenemia in PCOS women. The correlation results between VAI and FAI have not been published by other researchers as yet. Very few studies have evaluated the usefulness of VAI in PCOS women. Therefore, it is necessary to carry out reliable, large population-based studies among European women diagnosed with polycystic ovary syndrome.

\section{Conclusions}

Following the studies conducted by the authors of this manuscript, it may be concluded that utilizing VAI during routine medical examinations makes possible early prevention of metabolic disorders (including cardiometabolic disorders), as well as assessment of hyperandrogenemia severity in women with polycystic ovary syndrome. The cut-off point for VAI = 1.675 is an easy tool for evaluating women with metabolically unhealthy polycystic ovary syndrome (MU-PCOS).

\section{References}

1. Amato MC, Giordano C. Visceral Adiposity Index: An indicator of adipose tissue dysfunction. Int J Endocrinol. 2014, ID 730827, 7. doi: 10.1155/2014/730827.

2. Oh JY, Sung YA, Lee HJ. The visceral adiposity index as a predictor of insulin resistance in young women with polycystic ovary syndrome. Obesity. 2013;21:1690-1694. doi:10.1002/oby.20096.

3. Amato MC, Giordano C, Galia M, et al. AlkaMeSy Study Group: Visceral adiposity index: A reliable indicator of visceral fat function associated with cardiometabolic risk. Diabetes Care. 2010;33:920-922.

4. Ranasinha S, Joham AE, Norman RF, et al. The association between polycystic ovary syndrome (PCOS) and metabolic syndrome: A statistical modelling approach. Clinical Endocrinology. 2015;12830. doi: 10.1111/cen.12830.

5. Dunaif $A$. Insulin resistance and the polycystic ovary syndrome: Mechanism and implications for pathogenesis. Endocrine Reviews. 1997;18:774-800.

6. Amato MC, Guarnotta V, Forti D, Donatelli M, Dolcimascolo S, Giordano C. Metabolically healthy polycystic ovary syndrome (MH-PCOS) and metabolically unhealthy polycystic ovary syndrome (MU-PCOS): A comparative analysis of four simple methods useful for metabolic assessment. Human Reproduction. 2013;28:1919-1928.

7. Androulakis II, Kandaraki E, Christakou C, et al. Visceral adiposity index (VAI) is related to the severity of anovulation and other clinical features in women with polycystic ovary syndrome. Clin Endocrinol (Oxf). 2014;81:426-431.

8. Amato MC, Magistro A, Gambino G, Vesco R, Giordano C. Visceral adiposity index and DHEAS are useful markers of diabetes risk in women with polycystic ovary syndrome. Eur J Endocrinol. 2015;172:79-88. 